\title{
Ceruminous Adenocarcinoma
}

National Cancer Institute

\section{Source}

National Cancer Institute. Ceruminous Adenocarcinoma. NCI Thesaurus. Code C4176.

An infiltrating adenocarcinoma derived from ceruminous glands in the external auditory canal. 